\title{
LACTB and LC3 could serve as potential biomarkers of gastric cancer to neoadjuvant chemotherapy with oxaliplatin plus S-1
}

\author{
FANG YANG ${ }^{1-3^{*}}$, ZHIQIANG YAN ${ }^{4 *}$, WEI NIE ${ }^{2}$, ZEYING LIU $^{3}$, XINGZHEN CHENG $^{4}$, \\ WEI WANG ${ }^{3}$, CHUNYAN SHAO ${ }^{3}$, GUI FU $^{3}$ and YANNI YU ${ }^{1}$ \\ ${ }^{1}$ Department of Pathology; ${ }^{2}$ School of Clinical Laboratory Science, Guizhou Medical University; \\ ${ }^{3}$ Laboratory Department of Guizhou Cancer Hospital; ${ }^{4}$ Department of Gastrointestinal Surgery, \\ Affiliated Hospital of Guizhou Medical University, Guiyang, Guizhou 550004, P.R. China
}

Received November 13, 2020; Accepted March 17, 2021

DOI: $10.3892 / 01.2021 .12731$

\begin{abstract}
The present study investigated and evaluated the correlation between the expression of LACTB and LC3 and the clinical outcomes of patients with advanced gastric cancer treated with oxaliplatin plus S-1 neoadjuvant chemotherapy (NACT). A total of 51 patients with advanced gastric cancer underwent NACT treatment between June 2015 and June 2017. Pathomorphological changes in gastric cancer were analyzed by H\&E staining. The expression level and subcellular localization of LACTB and LC3 in paraffin-embedded biopsies were detected by immunohistochemistry and immunofluorescence. The mRNA and protein expression of LACTB were investigated by reverse transcription quantitative polymerase chain reaction and Western blotting, respectively. Statistical analysis was performed to determine the association between the expression of LACTB and LC3 and clinical chemotherapy efficacy of NACT for gastric cancer. Among the 51 patients, $3(5.88 \%), 27(52.94 \%), 13(25.49 \%)$ and 8 (15.69\%) displayed complete remission, partial remission, stable disease and progressive disease, respectively. The rate of decreased LACTB expression was $68.6 \%$, while the rate of increased LC3 expression was $60.8 \%$. Furthermore, there was a significant negative correlation between the expression of LACTB and that of LC3 following NACT $(\mathrm{P}<0.001)$. High expression of LC3 $(\mathrm{P}<0.01)$ and low expression of LACTB $(\mathrm{P}<0.01)$ were associated with a poor response of patients with advanced gastric cancer to NACT. In conclusion, the expression of LACTB and LC3 may serve as a promising novel biomarker for determining the prognosis of patients with advanced gastric cancer receiving
\end{abstract}

Correspondence to: Ms. Yanni Yu, Department of Pathology, Guizhou Medical University, 4 Beijing Road, Yunyan, Guiyang, Guizhou 550004, P.R. China

E-mail: gyyxybl2010@sina.com

${ }^{*}$ Contributed equally

Key words: gastric cancer, LACTB, LC3, neoadjuvant chemotherapy, prognosis, SOX
NACT, while its potential clinical significance requires further elucidation.

\section{Introduction}

Gastric cancer is one of the most common malignant tumor types in the digestive system, with a steadily increasing incidence in numerous countries. There are $\sim 1$ million new cases with gastric cancer reported each year (1). Early clinical manifestations of gastric cancer are mild and difficult to be diagnosed. Once the patients are diagnosed with relevant clinical manifestations, the majority have reached a stage with metastasis, thereby losing the best time for treatment (2). At present, neoadjuvant chemotherapy (NACT) has been found to have significant clinical efficacy in the treatment of solid tumors, including liver cancer, gastric cancer and breast cancer. In this case, NACT may decrease the risk of postoperative recurrence and metastasis, while improving the survival rate and quality of life of patients (3-5). However, not all patients respond to NACT due to the presence of a tumor resistance mechanism affecting the change in drug efficacy during treatment. Therefore, it is vital to select a reasonable treatment regimen and optimize the prognosis of patients to identify a reliable predictive index of chemotherapy sensitivity and early diagnosis of gastric cancer.

Autophagy is a process of cellular self-digestion under stress. In recent years, an increasing number of studies have demonstrated that autophagy serves an important role in the growth and differentiation of tumor cells $(6,7)$. The formation of autophagosomes requires the participation of various protein complexes and small molecules, including the hallmark protein LC3. LC3-mediated autophagy has been revealed to be critical in a variety of gastric diseases $(8,9)$. Mitochondrial gene beta-lactamases (LACTB) encoding a mitochondrial membrane protein may either inhibit cell proliferation by regulating the mitochondrial lipid metabolism and tumor cell state, or suppress tumor growth by directly binding to the tumor suppressor gene, P53 (10). It has been demonstrated that abnormal expression of LACTB was associated with obesity and atherosclerosis (11). Furthermore, the occurrence of gastric cancer has been proven to be associated with intracellular mechanisms underlying lipid accumulation 
in gastric epithelial neoplasms (12). These findings suggested that the association between LACTB and diseases associated with abnormal lipid metabolism has potential research value. We hypothesized that the expression of LACTB and LC3 may be associated with the occurrence and development of gastric cancer.

In the present study, the clinical data of 51 patients with advanced gastric cancer treated with NACT were analyzed, and the effect of NACT on the expression of LACTB and LC3 in gastric cancer tissues was investigated. The present study investigated the relevant efficacy and prognosis, thereby laying an evidence-based basis for the screening of chemotherapy regimens for gastric cancer.

\section{Materials and methods}

Patients and samples. A total of 51 patients with histologically-confirmed advanced gastric cancer were enrolled between June 2015 and June 2017 in the Department of Gastrointestinal Surgery of Guizhou Medical University (Guizhou, China). All patients underwent pretreatment clinical evaluation, including a complete medical history review, physical examination, gastroscopy, whole abdomen enhanced CT and tumor marker examination. These patients were aged between 34 and 80 years with a mean age of $61.67 \pm 12.18$ years and consisted of 35 males and 16 females. The following clinicopathological factors were recorded: Age, sex, tumor location, Borrmman classification (13), Tumor-Node-Metastasis (TNM), histological differentiation, response to NACT and LC3, and the expression level of LACTB. The TNM staging was defined according to the rules of the American Joint Commission on Cancer system manual, 7th edition (14). The Human Ethics Review Committee of the Affiliated Hospital of Guizhou Medical University approved the present study, and written informed consent was obtained from the patients for the use of their biopsy materials.

Eligibility criteria. Patients with histologically-confirmed locally advanced or metastatic gastric cancer were considered eligible for the present study. Furthermore, the patients met the following criteria: i) No other uncontrolled or severe primary malignant tumors or underlying disease; ii) aged between 20 and 80 years; and iii) fulfilling the selection criteria for preoperative NACT, as well as agreeing to undergo NACT.

Neoadjuvant chemotherapy. All patients in the present study underwent NACT treatment with the oxaliplatin plus S-1 (SOX) (cat. no. H20040817; Jiangsu Hengrui Pharmaceutical Co., Ltd.); oxaliplatin $\left(130 \mathrm{mg} / \mathrm{m}^{2}\right)$ was intravenously injected on day 1, while S-1 (50 mg bid) was administered orally from day 1 to day 14 . The aforementioned procedure was repeated every 3 weeks for 2-4 cycles.

Evaluation of efficacy. To determine whether patients could continue chemotherapy, they underwent routine blood and biochemical tests prior to the start of each chemotherapy cycle, including granulocyte, hemoglobin, platelet, liver function and kidney function tests. Following 2 cycles of chemotherapy, the patients were assessed by ultrasonic gastroscopy, whole abdomen enhanced CT examination and tumor marker, such as carcinoembryonic antigen, carbohydrate antigen 125 , carbohydrate antigen 199 monitoring. The following treatment options were applied based on the evaluation: Patients directly underwent surgery if the lesions had progressed; those with smaller lesions continued one cycle of NACT; those without significant improvement in the lesions communicated with their family members and voluntarily selected direct surgery or continued 1-2 cycles of chemotherapy.

NACT was conducted as mentioned earlier, and the tumor response to neoadjuvant treatment was reviewed using the Response Evaluation Criteria in Solid Tumors (15). A complete response (CR) and a partial response (PR) were defined as complete disappearance of the lesion and $>30 \%$ decrease in the maximum transverse diameter of the primary lesion, respectively. Additionally, cases with new lesions or $>20 \%$ increase in the maximum transverse diameter of primary lesions were evaluated as progressive disease (PD), while those who failed to meet these criteria were classified as having stable disease (SD).

Immunohistochemistry (IHC). The cancer tissue specimens were fixed in $10 \%$ formaldehyde at $4^{\circ} \mathrm{C}$ for $3-24 \mathrm{~h}$, embedded in paraffin, and continuously sectioned at a thickness of $4 \mu \mathrm{m}$. The specimens were attached to the slides treated with poly-L-lysine and baked at $80^{\circ} \mathrm{C}$ for $50 \mathrm{~min}$. Sections were incubated with $3 \%$ hydrogen peroxide for $10 \mathrm{~min}$ at room temperature to block the endogenous peroxidase activity. The tissue sections were then incubated with rabbit polyclonal antibodies against LACTB (dilution, 1:200; cat. no. ab244454; Abcam) and LC3 (dilution, 1:500; cat. no. ab63817; Abcam) overnight at $4{ }^{\circ} \mathrm{C}$, washed 3 times with phosphate buffered saline (PBS) containing $0.1 \%$ Tween-20, and exposed to the horseradish peroxidase-labeled goat anti-rabbit secondary antibody $\operatorname{IgG}(1: 2,000$; cat. no. ab6112; Abcam) for $30 \mathrm{~min}$ at $20^{\circ} \mathrm{C}$. Immunoreactions were detected by the DAKO REAL EnVision Detection System-HRP (Dako; Agilent Technologies, Inc.). The color reaction was performed using 3,3'-diaminobenzidine after incubation for $5 \mathrm{~min}$ at room temperature, and the sections were stained with hematoxylin and eosin (H\&E) for 5 and $3 \mathrm{~min}$ at room temperature, respectively. The images of IHC and H\&E staining were obtained using the OLYMPUS BX53 light microscope (magnification, $x 400$ ) (Olympus Corporation), and the digital slides were analyzed by the software Image J v.1.8.0 (National Institutes of Health). The expression and subcellular distribution of LACTB and LC3 in the slices were observed under a light microscope, and five high-power fields (magnification, x400) were selected for each slice. Staining intensity was categorized as follows: No staining, 0 points; light yellow, 1 point; dark yellow, 2 points; and brown or tan, 3 points. Additionally, the rate of positive staining cells was scored as: 0 points for $1<10 \%, 1$ point for $10-25 \%, 2$ points for $26-50 \%, 3$ points for $51-75 \%$, and 4 points for $>76 \%(16,17)$. The final score was then calculated by multiplying the staining intensity by the percentage of positive cells in staining areas. With the cut-off value of LACTB and LC3 as 5 and 3, respectively, a final score of greater than the value was defined as high expression, whereas that of less than the value was determined as low expression $(18,19)$. The staining was scored independently by two senior pathologists in a double-blinded manner. 
Table I. Clinicopathological factors and their association with the expression of LACTB and LC3 proteins in gastric cancer prior to neoadjuvant chemotherapy.

\begin{tabular}{|c|c|c|c|c|c|}
\hline \multirow[b]{2}{*}{ Clinical parameter } & \multirow[b]{2}{*}{ Number of cases, $\mathrm{n}$} & \multicolumn{2}{|c|}{ LACTB } & \multicolumn{2}{|c|}{ LC3 } \\
\hline & & Low & High & Low & High \\
\hline \multicolumn{6}{|l|}{ Sex } \\
\hline Male & 35 & 20 & 15 & 25 & 8 \\
\hline Female & 16 & 8 & 8 & 14 & 2 \\
\hline$\chi^{2}$ & & & 2.519 & & \\
\hline P-value & & & 0.112 & & 0.464 \\
\hline \multicolumn{6}{|l|}{ Age } \\
\hline$<65$ years & 24 & 14 & 10 & 20 & 4 \\
\hline$\geqq 65$ years & 27 & 14 & 13 & 21 & 6 \\
\hline$\chi^{2}$ & & & 0.216 & & \\
\hline P-value & & & 0.642 & & 0.731 \\
\hline \multicolumn{6}{|l|}{ Histological differentiation } \\
\hline Moderately-differentiated gastric adenocarcinoma & 16 & 13 & 3 & 12 & 4 \\
\hline Poorly-differentiated gastric adenocarcinoma & 35 & 28 & 7 & 29 & 6 \\
\hline P-value & & & 1.000 & & 0.705 \\
\hline \multicolumn{6}{|l|}{ Tumor location } \\
\hline Fundus & 6 & 3 & 2 & 5 & 1 \\
\hline Body & 9 & 5 & 4 & 7 & 2 \\
\hline Antral & 36 & 25 & 11 & 28 & 7 \\
\hline P-value & & & 0.316 & & 0.578 \\
\hline \multicolumn{6}{|l|}{ Borrmman type } \\
\hline I-II & 16 & 10 & 6 & 12 & 4 \\
\hline III-IV & 35 & 20 & 15 & 29 & 6 \\
\hline$\chi^{2}$ & & & 0.165 & & \\
\hline P-value & & & 0.685 & & 0.705 \\
\hline \multicolumn{6}{|l|}{ TNM stage } \\
\hline I-II & 7 & 2 & 5 & 5 & 2 \\
\hline III-IV & 44 & 26 & 18 & 36 & 8 \\
\hline P-value & & & 0.221 & & 0.612 \\
\hline
\end{tabular}

$\mathrm{P}>0.05$, no statistically significant difference. TNM, Tumor-Node-Metastasis.

Immunofluorescence. The tissues were rinsed with PBS 3 times for 5 min each, blocked with 5\% normal goat serum in a wet box for $30 \mathrm{~min}$ at room temperature, and then incubated with mouse monoclonal primary antibodies against LC3 (dilution, 1:100; cat. no. ab243506; Abcam) or LACTB (dilution, 1:100; cat. no. ab244455; Abcam) at $4^{\circ} \mathrm{C}$ for $24 \mathrm{~h}$. Following being rinsed 3 times with PBS, the tissues were incubated with FITC-labeled goat anti-mouse secondary antibody $\operatorname{IgG}$ (1:200; cat. no. ab150113; Abcam) at room temperature for $1 \mathrm{~h}$ in the dark, and then rinsed 3 times with PBS. No primary antibody was added to the negative control. The tissues were counterstained with DAPI for $5 \mathrm{~min}$ at room temperature, and images were captured under a fluorescence microscope (magnification, x400) (Leica AF6000 cell station). Images of the nucleus (DAPI staining, blue fluorescence) and cell body (LC3 or LACTB staining, red fluorescence) were captured separately, and the two images were merged for an overlapping image for counting of cells positively stained for LC3 or LACTB.

Western blotting. Tissues were lysed in RIPA protein lysis solution (Biouniquer Technology Co., Ltd.), and extracted protein was quantified using the BCA protein quantification method. A total of $45 \mathrm{~g}$ of protein mixed with SDS protein sampling buffer was separated by $8-15 \%$ SDS-PAGE and electro-transferred onto a PVDF membrane. Following being blocked with TBST blocking solution containing 5\% skimmed milk powder for $100 \mathrm{~min}$ at room temperature, the membrane was incubated with diluted anti-LACTB antibody (dilution, 1:1,000; Abcam) or anti-LC3 antibody (dilution, 1:1,000; MBL International Co.) overnight at $4^{\circ} \mathrm{C}$. On the following day, the membrane was washed with TBST and subjected to an incubation with HRP-labeled secondary antibody (dilution, 1:2,000; Beijing Kangwei Biological 


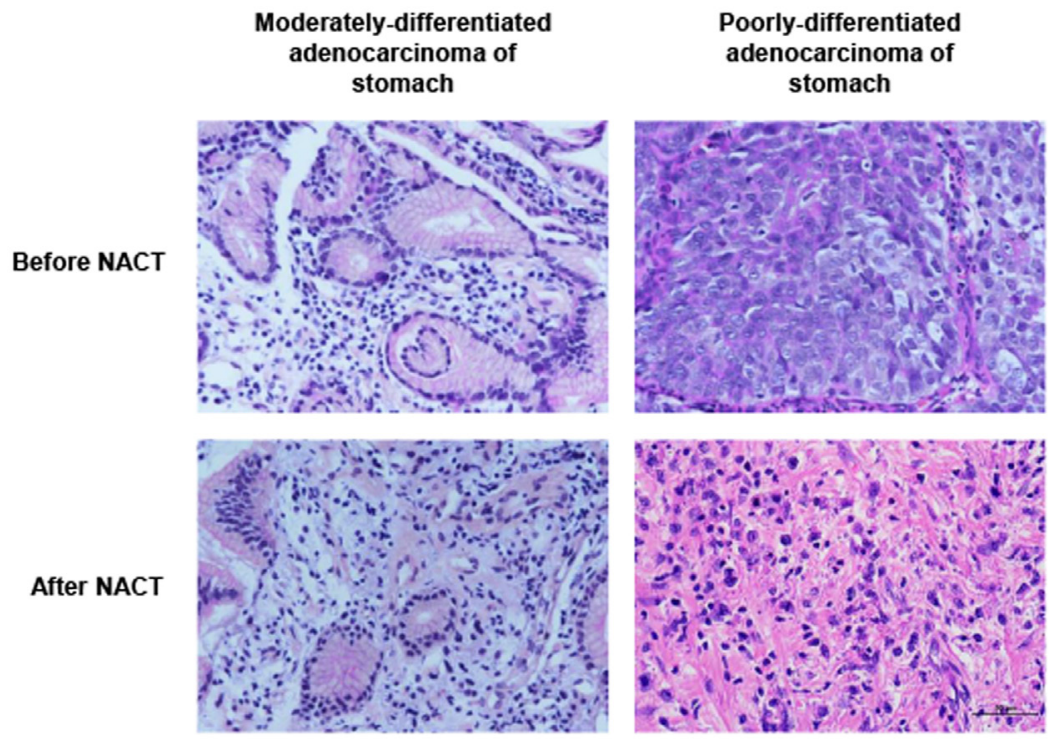

Figure 1. Histopathological changes in gastric cancer tissues following NACT with oxaliplatin plus S-1 (magnification, x400). NACT, neoadjuvant chemotherapy.

A

Before NACT

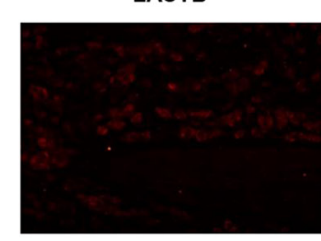

After NACT

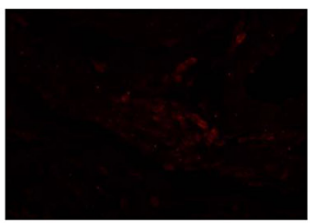

LC3

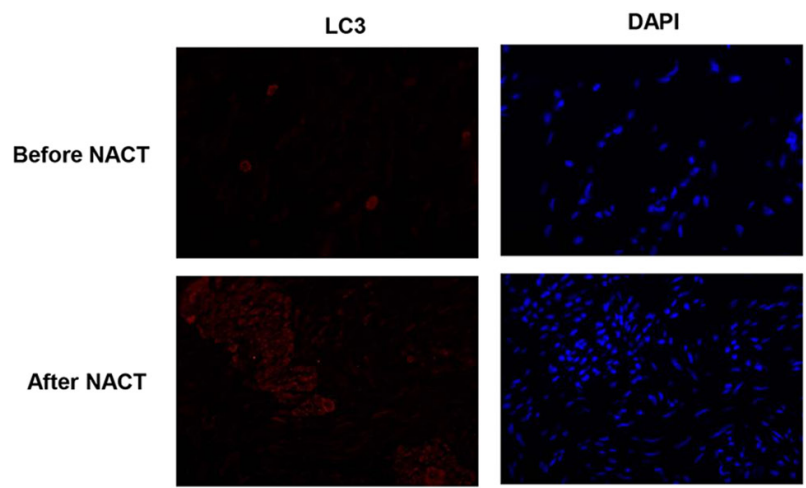

DAPI
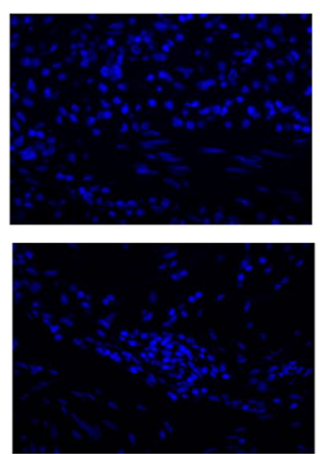

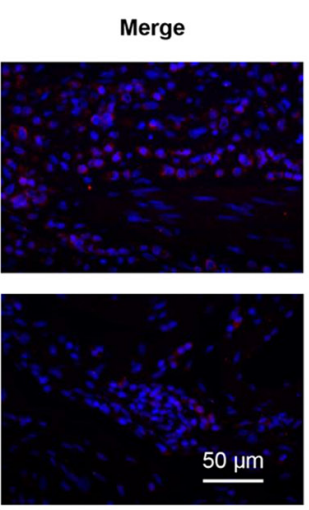

Merge

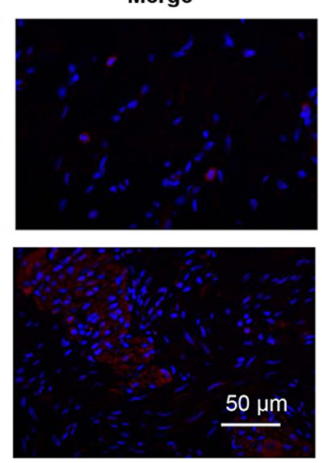

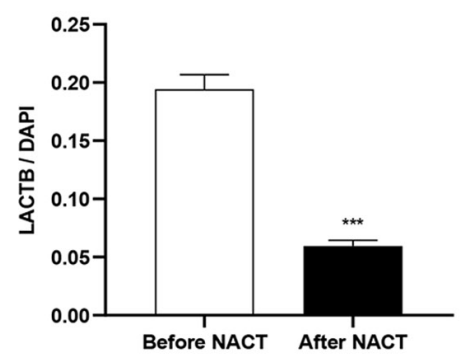

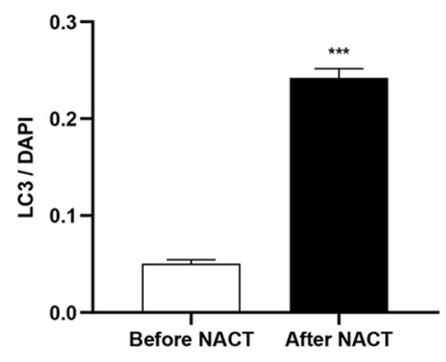

Figure 2. Immunofluorescence shows the distribution of (A) LACTB and (B) LC3 in gastric cancer tissues prior to and following NACT (magnification, $\mathrm{x} 400$ ). ${ }^{* * * *} \mathrm{P}<0.001$ vs. before NACT. NACT, neoadjuvant chemotherapy.

Technology Co., Ltd.) at room temperature for $1.5 \mathrm{~h}$. GAPDH was used as an internal control. Finally, target proteins in the membrane were exposed using enhanced chemiluminescence working fluid (Merck Millipore). The shaded value was calculated using Image Lab software v.4.0 (Bio-Rad Laboratories Inc.), and the ratio of the shaded value of LACTB and LC3 expression to that of GADPH expression was determined as the relative expression amount of the protein.
Reverse transcription-quantitative PCR (RT-qPCR). Total RNA was extracted from tissue using the TRIzol ${ }^{\circledR}$ reagent (Thermo Fisher Scientific, Inc.). Following identification of quality and purity, $2 \mu \mathrm{g}$ RNA was reverse transcribed into cDNA using SuperScript ${ }^{\circledR}$ III Reverse Transcriptase (Thermo Fisher Scientific, Inc.) at $42^{\circ} \mathrm{C}$ for $15 \mathrm{~min}$. Next, RT-qPCR was performed using SYBR Green I (Roche Diagnostics GmbH). GAPDH was used as the internal control. The primer sequences were as follows: GAPDH forward, 5'-CCTCGTCTCATAGAC 


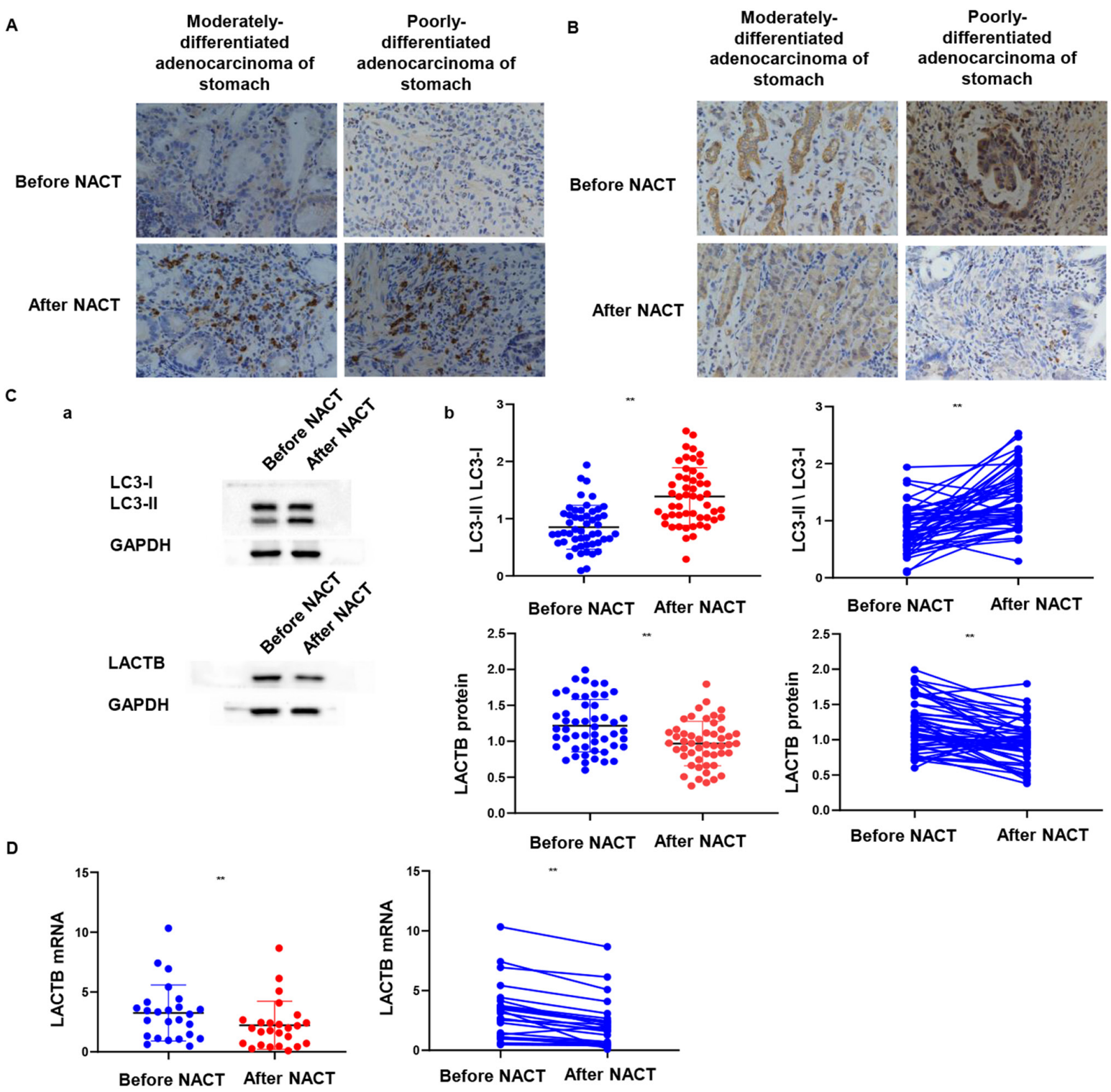

Figure 3. Effect of neoadjuvant therapy on the expression of LACTB and LC3 in gastric cancer tissues. Representative immunohistochemical staining images of (A) LACTB and (B) LC3 in gastric cancer tissues prior to and following neoadjuvant chemotherapy. (Ca) The protein expression of LACTB and LC3 was detected by western blotting. (Cb) Quantification of data presented in the panel. ${ }^{* *} \mathrm{P}<0.01$ vs. before NACT. (D) The mRNA expression of LACTB was determined by reverse transcription-quantitative polymerase chain reaction. Bars represent the median with the interquartile range. ${ }^{* *} \mathrm{P}<0.01$ as determined by Mann-Whitney U-test. NACT, neoadjuvant chemotherapy.

AAGATGGT-3' and reverse, 5'-GGGTAGAGTCATACT GGAACATG-3'; LACTB forward, 5'-CTGCTGCACAGG ATCAAGGA-3' and reverse, 5'-ATCCAGTTTCCCTGCTTC CC-3'. The following thermocycling conditions were applied: $95^{\circ} \mathrm{C}$ for $30 \mathrm{sec}$, followed by 50 cycles of denaturation at $95^{\circ} \mathrm{C}$ for $5 \mathrm{sec}$, and $60^{\circ} \mathrm{C}$ for $30 \mathrm{sec}$. At the end of the experiment, the cycle threshold $(\mathrm{Ct})$ value of each sample was obtained. Data were analyzed by using the comparative $2^{-\Delta \Delta C q}$ method (20).

Statistical analysis. Statistical analysis was performed using SPSS 21.0 (IBM Corp.). The significant associations between the expression of LACTB or LC3 and various clinicopathological parameters were determined by the Pearson's chi-square test or Fisher's exact test. Based on the normality of the distribution, the results are expressed as either the mean \pm standard deviation or the median and interquartile range. Differences between two groups were compared by the Student's t-test. The Mann-Whitney U test was used to investigate the difference between the mRNA expression of LACTB prior to and following NACT. The correlation between the expression of LACTB and LC3 prior to and following NACT was analyzed using the Spearman correlation coefficient. $\mathrm{P}<0.05$ was used to indicate a statistically significant difference.

\section{Results}

Clinical factors and their association with the expression of LACTB and LC3 proteins in gastric cancer prior to SOX regimen NACT. The clinicopathological data of 51 patients with gastric cancer were obtained, and their associations with the expression levels of LACTB and LC3 were analyzed. As summarized in Table I, there were no statistically significant 
Table II. Correlation between the expression of LACTB and LC3 prior to and following neoadjuvant chemotherapy.

\begin{tabular}{|c|c|c|c|c|c|c|}
\hline \multirow[b]{2}{*}{ LACTB } & \multirow[b]{2}{*}{$\mathrm{n}$} & \multicolumn{3}{|c|}{ LC3 } & \multirow[b]{2}{*}{$r_{s}$} & \multirow[b]{2}{*}{ P-value } \\
\hline & & Decreased & Invariant & Increased & & \\
\hline Decreased & 32 & 6 & 1 & 25 & & \\
\hline Invariant & 5 & 1 & 0 & 4 & -0.785 & 0.000 \\
\hline Increased & 14 & 10 & 2 & 2 & & \\
\hline
\end{tabular}

Table III. Difference between the expression of LACTB or LC3 in gastric cancer prior to and following neoadjuvant chemotherapy.

\begin{tabular}{|c|c|c|c|c|c|c|c|}
\hline \multirow[b]{2}{*}{ Protein } & \multirow[b]{2}{*}{ Number of cases (n) } & \multicolumn{4}{|c|}{ Clinical efficacy of chemotherapy } & \multirow[b]{2}{*}{$\chi^{2}$} & \multirow[b]{2}{*}{ P-value } \\
\hline & & CR & PR & SD & PD & & \\
\hline \multicolumn{8}{|l|}{ LACTB } \\
\hline Decreased & 32 & 0 & 13 & 12 & 7 & 17.648 & 0.007 \\
\hline Invariant & 5 & 1 & 2 & 1 & 1 & & \\
\hline Increased & 14 & 2 & 12 & 0 & 0 & & \\
\hline \multicolumn{8}{|l|}{ LC3 } \\
\hline Decreased & 17 & 3 & 13 & 1 & 0 & & 0.000 \\
\hline Invariant & 3 & 0 & 2 & 1 & 0 & & \\
\hline Increased & 31 & 0 & 12 & 11 & 8 & & \\
\hline
\end{tabular}

CR, complete remission; PR, partial remission; $\mathrm{SD}$, stable disease; PD, disease progression.

differences in the expression levels of LACTB and LC3 among different clinicopathological subgroups in terms of sex, age, histological differentiation, tumor location, Borrmman type and TNM stage $(\mathrm{P}>0.05)$.

Effect of SOX regimen NACT on the pathomorphology of gastric cancer. Prior to NACT, moderately-differentiated adenocarcinoma with glandular duct structure in the stomach can be observed, and the cancer cells were columnar or cubic in shape and arrangement. Poorly-differentiated gastric adenocarcinomas only displayed a tendency to form glandular duct structures. Cancer cells were short columnar, cubic or amorphous, forming strange nuclear cells, tumor giant cells or cancer cells with strip or scattered distribution, unclear nuclear structure and irregular shape, while pathological mitotic phases may be easily identified. By contrast, few cancer cells were identified in the interstitium in a strip-like or nest-like arrangement following NACT with SOX. As shown in Fig. 1, only a single cancer cell remained in well-differentiated gastric adenocarcinoma.

Effect of SOX regimen NACT on the expression distribution of $L A C T B$ and LC3 in gastric cancer tissues. The present study further analyzed the subcellular distribution of LACTB and LC3 protein in the gastric cancer cells by using immunofluorescence. As depicted in Fig. 2, LACTB and LC3 were mostly localized to the cytoplasm or cell membrane, exhibiting needle-tip-like signals. Following NACT, a significant decrease in LACTB expression was detected in the gastric cancer tissues; negative staining for LACTB was present in the majority of the gastric cancer tissues, while very little cytoplasmic LACTB expression was observed. By contrast, LC3 was highly expressed in the cytoplasm of gastric cancer cells following NACT.

Effect of SOX regimen NACT on the expression levels of $L A C T B$ and LC3 in gastric cancer. IHC revealed that while LACTB staining was visualized as brown-yellow granules localized to the cell membrane and cytoplasm (Fig. 3A), LC3 was stained positively as brown granules in the cytoplasm and occasionally in the nuclei of the cancer cells (Fig. 3B). Among 51 gastroscopic biopsy specimens of gastric cancer prior to SOX NACT, 29 (56.9\%) and 11 (21.6\%) were positive for LACTB and LC3 expression, respectively. Notably, following NACT, LACTB and LC3 were expressed in 14 (27.5\%) and 31 $(60.78 \%)$ gastric cancer specimens, respectively. Furthermore, Western blotting demonstrated that the expression of LACTB in gastric cancer tissues prior to NACT was significantly higher than that following the treatment. By contrast, LC3 protein expression was significantly increased during this period $(\mathrm{P}<0.01$; Fig. $3 \mathrm{C})$. To verify this observation, the mRNA expression level of LACTB was analyzed using RT-qPCR and it was revealed that LACTB expression levels in gastric cancer tissues following NACT were significantly lower than that prior to the treatment $(\mathrm{P}<0.01$; Fig. 3D).

The expression of LACTB and LC3 predicts response of gastric cancer to NACT with SOX. Among 51 patients undergoing NACT, 3 (5.88\%), 27 (52.94\%), 13 (25.49\%) and 8 (15.69\%) 
displayed CR, PR, SD and PD, respectively. While the rate of decreased LACTB expression was $68.6 \%$, the rate of increased LC3 expression was $60.8 \%$. As shown in Table II, there was a significant negative correlation between the expression of LACTB and LC3 prior to and following NACT $(\mathrm{P}<0.001)$. Furthermore, it was observed that the levels of LACTB and LC3 following NACT were significantly correlated with the curative effect of NACT $(\mathrm{P}<0.01 ; \mathrm{P}<0.01$; Table III).

\section{Discussion}

Gastric cancer is the second leading cause of cancer-related mortality worldwide, and is the third leading cause of cancer-related mortality in males and the second leading cause of cancer-related mortality in females in China (21). With the development of chemotherapy technology for gastric cancer, particularly the discovery of a series of novel therapeutic targets, a growing number of NACT regimens have been applied in the clinical treatment of gastric cancer. NACT with SOX is currently one of the commonly used chemotherapy regimens for the treatment of advanced gastric cancer (22). The present retrospective study demonstrated that, as a neoadjuvant regimen for advanced gastric cancer, SOX chemotherapy had a total effective rate (CR+PR) of $58.8 \%$, which was similar to that reported previously (23). However, in clinical practice, a considerable proportion of patients have tumor progression during chemotherapy and a delay in treatment, thereby affecting the overall efficacy. Therefore, molecular biomarkers are required for guiding neoadjuvant treatment. The present study reported that the expression of LACTB and LC3 in gastric cancer was significantly associated with the curative effect of NACT. To the best of our knowledge, the present study was the first to identify the role of LACTB and LC3 in gastric cancer with neoadjuvant chemotherapy.

The present study demonstrated that there was no correlation between the expression level of LACTB or LC3 proteins and clinicopathological indices of gastric cancer, including sex, age, histological differentiation, tumor location, Borrmman type and TNM stage. Based on the aforementioned findings, the expression of LC3 and LACTB was further investigated in the biopsies of 51 patients with advanced gastric cancer undergoing preoperative chemotherapy by immunohistochemistry and Western blotting. The staining revealed that LC3 and LACTB are mainly localized in the cytoplasm. The aforementioned observations are consistent with the subcellular localization of LC3 in resected gastrointestinal cancer tissues reported in a previous study (24). Following NACT, LC3 was significantly expressed in 31 cases $(60.78 \%)$, while LACTB was only significantly expressed in 14 cases of gastric cancer (27.5\%). Previous studies have reported that the autophagy activity of numerous malignant tumor types is lower than that of normal tissues, while radiation or antitumor drugs may increase autophagy activity and induce autophagic death of tumor cells $(25,26)$. Tumor resistance to anticancer therapies, including chemotherapy and radiation therapy, may be enhanced through upregulation of transcription of autophagy-inducible factors (27). LC3 is divided into two subtypes, type I LC3 (LC3-I) and type II LC3 (LC3-II). In the physiological state, LC3 synthesized in cells is routinely processed into cytoplasmic soluble LC3-I. Upon autophagy induction, LC3-I undergoes a ubiquitin-like modification to bind with phosphatidylethanolamine on the surface of the autophagy membrane to form LC3-II. Therefore, the ratio of LC3-II/LC3-I is an important index to evaluate the occurrence and level of autophagy. Clinicopathological studies of liver tumors have demonstrated that LC3 expression may serve as an important prognostic factor for hepatocellular carcinoma, particularly for those patients undergoing surgical resection (28). The association between increased LC3 expression and a poor prognosis in gastric cancer has been extensively studied (29-31). All these studies have indicated that LC3 may be used as an independent prognostic indicator for patients with gastric cancer. As a tumor suppressor, LACTB is highly expressed in numerous solid tumor types. Zhang et al (32) reported that downregulation of LACTB was significantly associated with a poor clinical prognosis in breast cancer. Furthermore, Li et al (33) demonstrated that LACTB-overexpression in glioma cells may inhibit cell proliferation, invasion and angiogenesis, while low expression of LACTB was associated with a poor prognosis in patients with glioma. Furthermore, it has been demonstrated that patients with colorectal cancer with a low expression of LACTB have a poor overall prognosis (34).

Previous studies have demonstrated that the main obstacle to the development of PD during chemotherapy is multidrug resistance $(35,36)$. The development of multidrug resistance will lead to an increase in cancer-associated mortality. The resistance to NACT in triple-negative breast cancer, ovarian cancer and bladder cancer has been reported in previous studies (37-39). In the present study, among 51 patients with advanced gastric cancer undergoing NACT, 36 displayed an increase in the expression level of LC3; of the 36 patients, 8 developed PD. Meanwhile, 29 out of 51 patients receiving the treatment had a decrease in the expression level of LACTB; 6 of the 29 patients had PD. Notably, 2 of the 6 patients with unchanged expression level of LACTB following NACT developed PD. Numerous factors are associated with chemoresistance. However, the expression of resistance genes is obtained by reprogramming following chemotherapy. In addition, the development of drug resistance may be associated with the dose of chemotherapeutic drugs, course of treatment and treatment regimen.

Taken together, these data suggested that certain patients with gastric cancer undergoing NACT may develop multidrug resistance, and abnormal expression of LACTB and LC3 may indicate a poor prognosis, leading to resistance to NACT. Further studies are required to investigate the association between the expression of LACTB or LC3 and the drug resistance of gastric cancer cells, as well as the molecular mechanism underlying the involvement of LACTB in the occurrence and development of gastric cancer. Furthermore, given the small number of patients, the absence of a retrospective design and survival analysis and the presence of confounding factors, including environmental factors, in the present study, further validation of the findings is required.

In conclusion, the present study revealed a correlation between the expression level of LACTB and LC3 following NACT in gastric cancer and the efficacy of clinical chemotherapy, suggesting that the expression of LACTB and LC3 
may potentially serve as a prognostic factor for patients with gastric cancer.

\section{Acknowledgements}

Not applicable.

\section{Funding}

The present study was funded by the Guizhou Provincial Department of Education Project [grant no. YJSCXJH (2019) 069] and the Hospital-Level Project of Guizhou Cancer Hospital (grant no. YT2019019).

\section{Availability of data and materials}

The datasets used and/or analyzed during the current study are available from the corresponding author on reasonable request.

\section{Authors' contributions}

FY was responsible for the conception and design of the study. $\mathrm{ZY}$ was responsible for the collection and entry of data. WN, $\mathrm{ZL}, \mathrm{ZC}$ and WW were responsible for the collection and assembly of data. CS organized the data. GF was responsible for the experimental operations. YY was responsible for funding and project design. FY and ZY confirm the authenticity of all the raw data. All authors read and approved the final manuscript and agree to be accountable for all aspects of the research in ensuring that the accuracy or integrity of any part of the work are appropriately investigated and resolved.

\section{Ethics approval and consent to participate}

Ethical approval for the present study was provided by the Ethics Committee of the Affiliated Hospital of Guizhou medical university (Guizhou, China). All patients provided written informed consent for participations.

\section{Patient consent for publication}

Not applicable.

\section{Competing interests}

The authors declare that they have no competing interests.

\section{References}

1. Lott PC and Carvajal-Carmona LG: Resolving gastric cancer aetiology: An update in genetic predisposition. Lancet Gastroenterol Hepatol 3: 874-883, 2018

2. Tan Z: Recent advances in the surgical treatment of advanced gastric cancer: A review. Med Sci Monit 25: 3537-3541, 2019.

3. Derks MGM and van de Velde CJH: Neoadjuvant chemotherapy in breast cancer: More than just downsizing. Lancet Oncol 19: 2-3, 2018

4. Das M: Neoadjuvant chemotherapy: Survival benefit in gastric cancer. Lancet Oncol 18: e307, 2017.

5. Akateh C, Black SM, Conteh L, Miller ED, Noonan A, Elliott E, Pawlik TM, Tsung A and Cloyd JM: Neoadjuvant and adjuvant treatment strategies for hepatocellular carcinoma. World J Gastroenterol 25: 3704-3721, 2019.
6. Wei X, Duan W, Li Y, Zhang S, Xin X, Sun L, Gao M, Li Q and Wang D: AT101 exerts a synergetic efficacy in gastric cancer patients with 5-FU based treatment through promoting apoptosis and autophagy. Oncotarget 7: 34430-34441, 2016.

7. Liu M, Li CM, Chen ZF, Ji R, Guo QH, Li Q, Zhang HL and Zhou YN: Celecoxib regulates apoptosis and autophagy via the PI3K/Akt signaling pathway in SGC-7901 gastric cancer cells. Int J Mol Med 33: 1451-1458, 2014.

8. Masuda GO, Yashiro M, Kitayama K, Miki Y, Kasashima H, Kinoshita H,Morisaki T, Fukuoka T, Hasegawa T, Sakurai K, et al: Clinicopathological correlations of autophagy-related proteins LC3, beclin 1 and p62 in gastric cancer. Anticancer Res 36: 129-136, 2016.

9. Shida M, Kitajima Y, Nakamura J, Yanagihara K, Baba K, Wakiyama K and Noshiro H: Impaired mitophagy activates $\mathrm{mtROS} / \mathrm{HIF}-1 \alpha$ interplay and increases cancer aggressiveness in gastric cancer cells under hypoxia. Int J Oncol 48: 1379-1390, 2016.

10. Pascual G, Avgustinova A, Mejetta S, Martín M, Castellanos A, Attolini CS, Berenguer A, Prats N, Toll A, Hueto JA, et al: Targeting metastasis-initiating cells through the fatty acid receptor CD36. Nature 541: 41-45, 2017.

11. Lu JB, Yao XX, Xiu JC and Hu YW: MicroRNA-125b-5p attenuates lipopolysaccharide-induced monocyte chemoattractant protein-1 production by targeting inhibiting LACTB in THP-1 macrophages. Arch Biochem Biophys 590: 64-71, 2016.

12. Enjoji M, Kohjima M, Ohtsu K, Matsunaga K, Murata Y, Nakamuta M, Imamura K, Tanabe H, Iwashita A, Nagahama T and Yao K: Intracellular mechanisms underlying lipid accumulation (white opaque substance) in gastric epithelial neoplasms: A pilot study of expression profiles of lipid-metabolism-associated genes. J Gastroenterol Hepatol 31: 776-781, 2016.

13. Kim JH, Lee HH, Seo HS, Jung YJ and Park CH: Borrmann type 1 cancer is associated with a high recurrence rate in locally advanced gastric cancer. Ann Surg Oncol 25: 2044-2052, 2018.

14. Edge SB BD, Compton CC, Fritz AG, Greene FL and Trotti A (eds): AJCC cancer staging manual. 7th edition. Springer, New York, NY, 2010.

15. Armato SG III and Nowak AK: Revised modified response evaluation criteria in solid tumors for assessment of response in malignant pleural mesothelioma (version 1.1). J Thorac Oncol 13: 1012-1021, 2018.

16. Jaffer S, Orta L, Sunkara S, Sabo E and Burstein DE: Immunohistochemical detection of antiapoptotic protein $\mathrm{X}$-linked inhibitor of apoptosis in mammary carcinoma. Hum Pathol 38: 864-870, 2007.

17. Park JM, Huang S, Wu TT, Foster NR and Sinicrope FA: Prognostic impact of Beclin 1, p62/sequestosome 1 and LC3 protein expression in colon carcinomas from patients receiving 5 -fluorouracil as adjuvant chemotherapy. Cancer Biol Ther 14: 100-107, 2013.

18. Xue C, He Y, Zhu W, Chen X, Yu Y, Hu Q, Chen J, Liu L, Ren F, Ren Z, et al: Low expression of LACTB promotes tumor progression and predicts poor prognosis in hepatocellular carcinoma. Am J Transl Res 10: 4152-4162, 2018.

19. Wang J, Pan XL, Ding LJ, Liu DY, Da-Peng L and Jin T: Aberrant expression of beclin-1 and LC3 correlates with poor prognosis of human hypopharyngeal squamous cell carcinoma. PLoS One 8: e69038, 2013.

20. Papachristopoulou G, Tsapralis N, Michaelidou K, Ardavanis-Loukeris G, Griniatsos I, Scorilas A and Talieri M: Human kallikrein-related peptidase 12 (KLK12) splice variants discriminate benign from cancerous breast tumors. Clin Biochem 58: 78-85, 2018.

21. Ferlay J, Shin HR, Bray F and Mathers C: GLOBOCAN 2008, cancer incidence and mortality worldwide: IARC CancerBase no. 10. International Agency for Research on Cancer, Lyon, 2010.

22. Yamada Y, Higuchi K, Nishikawa K, Gotoh M, Fuse N, Sugimoto N, Nishina T, Amagai K, Chin K, Niwa Y, et al: Phase III study comparing oxaliplatin plus S-1 with cisplatin plus $\mathrm{S}-1$ in chemotherapy-naive patients with advanced gastric cancer. Ann Oncol 26: 141-148, 2015.

23. Li T and Chen L: Efficacy and safety of SOX regimen as neoadjuvant chemotherapy for advanced gastric cancer. Zhonghua Wei Chang Wai Ke Za Zhi 14: 104-106, 2011 (In Chinese).

24. Yoshioka A, Miyata H, Doki Y, Yamasaki M, Sohma I, Gotoh K, Takiguchi S, Fujiwara Y, Uchiyama Y and Monden M: LC3, an autophagosome marker, is highly expressed in gastrointestinal cancers. Int J Oncol 33: 461-468, 2008. 
25. Kimmelman AC and White E: Autophagy and tumor metabolism. Cell Metab 25: 1037-1043, 2017.

26. Amaravadi R, Kimmelman AC and White E: Recent insights into the function of autophagy in cancer. Genes Dev 30: 1913-1930, 2016.

27. $\mathrm{Hu}$ YL, Jahangiri A, Delay M and Aghi MK: Tumor cell autophagy as an adaptive response mediating resistance to treatments such as antiangiogenic therapy. Cancer Res 72: 4294-4299, 2012.

28. Lee YJ, Hah YJ, Kang YN, Kang KJ, Hwang JS, Chung WJ, Cho KB, Park KS, Kim ES, Seo HY, et al: The autophagy-related marker LC3 can predict prognosis in human hepatocellular carcinoma. PLoS One 8: e81540, 2013.

29. Wang X, Wu WKK, Gao J, Li Z, Dong B, Lin X, Li Y, Li Y, Gong J, Qi C, et al: Autophagy inhibition enhances PD-L1 expression in gastric cancer. J Exp Clin Cancer Res 38: 140, 2019.

30. Cao Y, Luo Y, Zou J, Ouyang J, Cai Z, Zeng X, Ling H and Zeng T: Autophagy and its role in gastric cancer. Clin Chim Acta 489: 10-20, 2019.

31. Kim JS, Bae GE, Kim KH, Lee SI, Chung C, Lee D, Lee TH Kwon IS and Yeo MK: Prognostic significance of LC3B and p62/SQSTM1 expression in gastric adenocarcinoma. Anticancer Res 39: 6711-6722, 2019.

32. Zhang J, He Y, Yu Y, Chen X, Cui G, Wang W, Zhang X, Luo Y, $\mathrm{Li}$ J, Ren F, et al: Upregulation of miR-374a promotes tumor metastasis and progression by downregulating LACTB and predicts unfavorable prognosis in breast cancer. Cancer Med 7: $3351-3362,2018$
33. Li HT, Dong DY, Liu Q, Xu YQ and Chen L: Overexpression of LACTB, a mitochondrial protein that inhibits proliferation and invasion in glioma cells. Oncol Res 27: 423-429, 2019.

34. Zeng K, Chen X, Hu X, Liu X, Xu T, Sun H, Pan Y, He B and Wang S: LACTB, a novel epigenetic silenced tumor suppressor, inhibits colorectal cancer progression by attenuating MDM2-mediated p53 ubiquitination and degradation. Oncogene 37: 5534-5551, 2018.

35. Yan C and Li TS: Dual role of mitophagy in cancer drug resistance. Anticancer Res 38: 617-621, 2018.

36. Cichocka-Radwan A and Lelonek M: Annual prognostic factors in chronic heart failure in patients over 80 years old. Kardiol Pol 75: 164-173, 2017.

37. Echeverria GV, Ge Z, Seth S, Zhang X, Jeter-Jones S, Zhou X, Cai S, Tu Y, McCoy A, Peoples M, et al: Resistance to neoadjuvant chemotherapy in triple-negative breast cancer mediated by a reversible drug-tolerant state. Sci Transl Med 11: eaav0936, 2019.

38. Glasgow MA, Argenta P, Abrahante JE, Shetty M, Talukdar S, Croonquist PA, Khalifa MA and Starr TK: Biological insights into chemotherapy resistance in ovarian cancer. Int J Mol Sci 20: 2131, 2019.

39. Buttigliero C, Tucci M, Vignani F, Scagliotti GV and Di Maio M: Molecular biomarkers to predict response to neoadjuvant chemotherapy for bladder cancer. Cancer Treat Rev 54: 1-9, 2017.

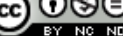

This work is licensed under a Creative Commons Attribution-NonCommercial-NoDerivatives 4.0 International (CC BY-NC-ND 4.0) License. 